University of Wollongong

Research Online

Faculty of Education - Papers (Archive)

Faculty of Arts, Social Sciences \& Humanities

2012

\title{
Directives canadiennes en matière d'activité physique pour la petite enfance (enfants âgés de 0 à 4 ans)
}

\author{
Mark S. Tremblay \\ Université d'Ottawa, Canada \\ Allana G. LeBlanc \\ Université d'Ottawa, Canada \\ Valerie Carson \\ Université Queen's, Canada \\ Louise Choquette \\ Centre de ressources Meilleur départ, Canada \\ Sarah Connor Gorber \\ Agence de santé publique du Canada
}

See next page for additional authors

Follow this and additional works at: https://ro.uow.edu.au/edupapers

Part of the Education Commons

\section{Recommended Citation}

Tremblay, Mark S.; LeBlanc, Allana G.; Carson, Valerie; Choquette, Louise; Gorber, Sarah Connor; Dillman, Carrie; Duggan, Mary; Gordon, Mary Jane; Hicks, Audrey; Janssen, Ian; Kho, Michelle E.; Latimer-Cheung, Amy E.; LeBlanc, Claire; Murumets, Kelly; Okely, Anthony D.; Reilly, John J.; Spence, John C.; Stearns, Jodie A.; and Timmons, Brian W.: Directives canadiennes en matière d'activité physique pour la petite enfance (enfants âgés de 0 à 4 ans) 2012, 357-369.

https://ro.uow.edu.au/edupapers/520

Research Online is the open access institutional repository for the University of Wollongong. For further information contact the UOW Library: research-pubs@uow.edu.au 


\section{Authors}

Mark S. Tremblay, Allana G. LeBlanc, Valerie Carson, Louise Choquette, Sarah Connor Gorber, Carrie Dillman, Mary Duggan, Mary Jane Gordon, Audrey Hicks, Ian Janssen, Michelle E. Kho, Amy E. LatimerCheung, Claire LeBlanc, Kelly Murumets, Anthony D. Okely, John J. Reilly, John C. Spence, Jodie A. Stearns, and Brian W. Timmons 


\title{
Directives canadiennes en matière d'activité physique pour la petite enfance (enfants âgés de 0 à 4 ans)
}

\author{
Mark S. Tremblay, Allana G. LeBlanc, Valerie Carson, Louise Choquette, \\ Sarah Connor Gorber, Carrie Dillman, Mary Duggan, Mary Jane Gordon, \\ Audrey Hicks, lan Janssen, Michelle E. Kho, Amy E. Latimer-Cheung, \\ Claire LeBlanc, Kelly Murumets, Anthony D. Okely, John J. Reilly, John C. Spence, \\ Jodie A. Stearns et Brian W. Timmons
}

\begin{abstract}
Résumé : La Société canadienne de physiologie de l'exercice (SCPE) en collaboration avec plusieurs partenaires, parties prenantes et chercheurs a élaboré de nouvelles Directives canadiennes en matière d'activité physique pour la petite enfance (enfants âgés de 0 à 4 ans). Ces directives nationales répondent à une demande pressante des praticiens en santé publique, en soins de santé, en pédiatrie et en condition physique désireux de promouvoir une vie active saine dès les premières années de la vie. L'élaboration complète des directives a respecté la Grille II d'évaluation de la qualité des recommandations pour la pratique clinique (AGREE) et la qualité des données a été évaluée au moyen de la méthodologie GRADE (Grading of Recommendations Assessment, Development and Evaluation). Les recommandations présentées dans cet article sont basées sur les données probantes d'une analyse documentaire systématique portant sur les relations entre l'activité physique et des indicateurs de santé (masse corporelle saine, santé des os et du squelette, développement des habiletés motrices, santé psychosociale, développement cognitif et facteurs de risque de maladie cardiométabolique) chez trois groupes d'âge (nourrissons $<1$ an, tout-petits âgés de 1-2 ans, enfants d'âge préscolaire, 3-4 ans). Les nouvelles directives sont composées d'un préambule situant le contexte et de directives spécifiques. Les directives finales ont bénéficié des fruits d'une vaste consultation en ligne auprès de plus de 900 intervenants concernés, d'utilisateurs finaux et de sources de premier plan, sur la scène nationale et internationale. La directive finale énonce ce qui suit : pour favoriser une croissance et un développement sains, les nourrissons (âgés de $<1$ an) devraient être physiquement actifs plusieurs fois par jour, particulièrement par l'entremise de jeux interactifs au sol. Les tout-petits (âgés de 1-2 ans) et les enfants d'âge scolaire (âgés de 3-4 ans) devraient être physiquement actifs chaque jour, quelle que soit l'intensité, pendant au moins 180 min réparties sur toute la journée, cela devrait comprendre une variété d'activités dans divers environnements à différentes intensités, des activités qui permettent de développer les habiletés motrices et une progression vers au moins $60 \mathrm{~min}$ de jeu actif à l'âge de 5 ans. S'adonner chaque jour à encore plus d'activité physique entraîne plus de bienfaits.
\end{abstract}

Reçu le 6 février 2012. Acceptée le 7 février 2012. Publié au www.nrcresearchpress.com/apnm, le 27 mars 2012.

M.S. Tremblay. Groupe de recherche sur les saines habitudes de vie et l'obésité, Institut de recherche du CHEO, Université d'Ottawa, 401, rue Smyth, Ottawa, ON K1H 8L1, Canada; Département de pédiatrie, Université d'Ottawa, 401, rue Smyth, Ottawa, ON K1H 8L1, Canada.

A.G. LeBlanc. Groupe de recherche sur les saines habitudes de vie et l'obésité, Institut de recherche du CHEO, Université d'Ottawa, 401, rue Smyth, Ottawa, ON K1H 8L1, Canada.

V. Carson et A.E. Latimer-Cheung. School of Kinesiology and Health Studies, Université Queen's, Kingston, ON K7L 3N6, Canada.

L. Choquette. Centre de ressources Meilleur départ, Nexus Santé, 180, rue Dundas Ouest, bureau 301, Toronto, ON M5G 1Z8, Canada.

S. Connor Gorber. Agence de santé publique du Canada, Ottawa, ON K1A 0K9, Canada.

C. Dillman et A. Hicks. Department of Kinesiology, Université McMaster, Hamilton, ON L8S 4K1, Canada.

M. Duggan. Société canadienne de physiologie de l'exercice, 18, rue Louisa, bureau 370, Ottawa, ON K1R 6Y6, Canada.

M.J. Gordon. KFL\&A Public Health, 221, av. Portsmouth, Kingston, ON K7M 1V5, Canada.

I. Janssen. School of Kinesiology and Health Studies and Department of Community Health and Epidemiology, Université Queen's, Kingston, ON K7L 3N6, Canada.

M.E. Kho. Department of Physical Medicine \& Rehabilitation, Johns Hopkins University, Baltimore, MD 21287, USA.

C. LeBlanc. L'Hôpital de Montréal pour enfants, 2300, rue Tupper, Montréal, QC H3H 1P3, Canada.

K. Murumets. ParticipACTION, 2, rue Bloor Est, bureau 1804, Toronto, ON M4W 1A8, Canada.

A.D. Okely. Faculty of Education, University of Wollongong, Wollongong, NSW 2522, Australia.

J.J. Reilly. Physical Activity for Health Group, School of Psychological Sciences and Health, University of Strathclyde, Jordanhill Campus, Glasgow, G13 1PP Scotland.

J.C. Spence et J.A. Stearns. Faculty of Physical Education and Recreation, Université de l'Alberta, Edmonton, AB T6G 2H9, Canada. B.W. Timmons. Child Health \& Exercise Medicine Program, Department of Pediatrics, Université McMaster, 1280, rue Main Ouest, Hamilton, ON L8S 4K1, Canada.

Auteur correspondant: Mark S. Tremblay (Courriel: mtremblay@cheo.on.ca). 
Mots-clés : activité physique, recommandations, directives, mesures, enfants, nourrissons, petite enfance, jeu, enfants d'âge préscolaire.

\begin{abstract}
The Canadian Society for Exercise Physiology (CSEP), with assistance from multiple partners, stakeholders and researchers, developed the first Canadian Physical Activity Guidelines for the Early Years (aged 0-4 years). These national guidelines were created in response to an urgent call from public health, health care, child care and fitness practitioners for healthy active living guidance for the early years. The guideline development process was informed by the Appraisal of Guidelines for Research Evaluation (AGREE) II instrument and the evidence assessed using the Grading of Recommendations Assessment, Development and Evaluation (GRADE) system. The recommendations are informed by evidence from a systematic review that examined the relationships between physical activity and health indicators (healthy body weight, bone and skeletal health, motor skill development, psychosocial health, cognitive development and cardio-metabolic disease risk factors) for three age groups (infants aged $<1$ year; toddlers aged 1-2 years; preschoolers aged 3-4 years). The new guidelines include a preamble to provide context, followed by the specific recommendations. The final guidelines benefitted from an extensive on-line consultation process with input from over 900 domestic and international stakeholders, end-users and key informants. The final guideline recommendations state that for healthy growth and development, infants (aged $<1$ year) should be physically active several times daily - particularly through interactive floor-based play. Toddlers (aged 1-2 years) and preschoolers (aged 3-4 years) should accumulate at least $180 \mathrm{~min}$ of physical activity at any intensity spread throughout the day, including a variety of activities in different environments, activities that develop movement skills, and progression toward at least $60 \mathrm{~min}$ of energetic play by 5 years of age. More daily physical activity provides greater benefits.
\end{abstract}

Key words: physical activity, recommendations, guidelines, measurement, children, infants, early years, play, preschoolers.

\section{Introduction et contexte}

Les études sur les enfants d'âge scolaire au Canada révèlent, preuve à l'appui, la prévalence sans précédent de l'obésité (Shields 2006; Tremblay and Willms 2000 ; Tremblay et al. 2002), le déclin de la condition physique (Tremblay et al. $2010 b$ ) et la faible pratique de l'activité physique (Colley et al. 2011). Le degré d'obésité est même élevé durant la petite enfance (Canning et al. 2004; Shields 2006). Dans la présente étude, la petite enfance est comprise entre 0 et 4 ans, soit de la naissance à la fin de la quatrième année (4,99 ans). Il est bien admis que la pratique régulière de l'activité physique est une mesure préventive efficace non seulement de l'obésité, mais aussi de divers troubles de santé chez des enfants d'âge scolaire (Janssen 2007; Janssen et LeBlanc 2010; Physical Activity Guidelines Advisory Committee 2008; World Health Organization 2010), mais les données probantes concernant les bienfaits sanitaires de l'activité physique au cours de la petite enfance ne sont pas claires (Timmons et al. 2007).

Entre 1998 et 2002, Santé Canada et la Société canadienne de physiologie de l'exercice (SCPE) ont publié les premières directives en matière d'activité physique à l'intention des enfants d'âge scolaire, des jeunes, des adultes et des personnes âgées, et ce, afin de mettre en place des objectifs mesurables à des fins de surveillance, de procurer des directives aux professionnels de la santé publique et pour motiver les Canadiennes et les Canadiens à être plus actifs (Tremblay et al. 2007a). Sous la gouverne de la SCPE, le processus d'élaboration de nouvelles directives (mises à jour) démarra en 2006 et aboutit à la publication de nouvelles directives en matière d'activité physique à l'intention des enfants d'âge scolaire (5-11 ans), des jeunes (12-17 ans), des adultes (18-64 ans) et des personnes âgées (65 ans et plus) au mois de janvier 2011 (Tremblay et al. 2011a). Pour plus d'information au sujet du processus ayant mené à l'élaboration de ces nouvelles directives, veuillez consulter les articles suivants (Tremblay et al. 2007a, 2007b, 2010a, 2011a) qui ont impliqué des experts en physiologie de l'exercice, dans les aspects psychosociaux de l'activité physique, en marketing social, en épidémiologie, en pédiatrie, en gérontologie, en santé publique, en conditionnement physique et dans l'élaboration de directives en matière d'activité physique. En plus de l'élaboration de nouvelles directives (mises à jour), on a identifié de nouvelles problématiques notamment en ce qui concerne la nécessité d'élaborer des directives en matière d'activité physique pour la petite enfance et d'autres tranches de la population (Autochtones, personnes présentant des incapacités, femmes enceintes, CSEP 2009; Tremblay et al. 2011a).

La nécessité de directives pour la petite enfance est évidente selon Timmons et al. (2007); d'après les statistiques d'accès aux périodiques, il y a 2 à 5 fois plus de téléchargement (près de 6000) de l'article de Timmons et al. (2007) que tout autre article de fond ayant servi à la mise à jour des directives (Tremblay et al. 2007b). De plus, au cours du processus de consultation pour la mise à jour des directives en matière d'activité physique à l'intention des enfants d'âge scolaire, des jeunes, des adultes et des personnes âgées sous la gouverne de la SCPE (CSEP 2011) et de l'Agence de santé publique du Canada, nous avons pris note de la forte demande de directives en matière d'activité physique à pour la petite enfance. En dernier lieu, la publication récente en Australie (Australian Government 2010) et au Royaume-Uni (Start Active Stay Active 2011) de directives en matière d'activité physique pour la petite enfance nous a incités à combler ce vide au Canada et à répondre à la demande claire de directives de la part des professionnels de la santé, des fournisseurs de soins de santé et du secteur de la petite enfance. Jusqu'à aujourd'hui, les chercheurs canadiens devaient utiliser les directives américaines pour évaluer les degrés de pratique de l'activité physique des enfants d'âge préscolaire (Obeid et al. 2011; Temple et al. 2009; Tucker 2008). 
Cet article présente les grandes lignes du processus et les résultats de l'élaboration des premières directives canadiennes en matière d'activité physique à l'intention des enfants (âgés de 0 à 4 ans) et qui ont été publiées par la SCPE au mois de mars 2012. Ces nouvelles directives résultent d'un processus rigoureux et transparent et les recommandations sont basées sur une analyse documentaire systématique des données probantes, un consensus d'experts et l'avis d'intervenants concernés par ce projet. Le rapport détaillé présentant en entier la méthodologie d'élaboration des directives et autres documents annexes est disponible sur le site Internet de la SCPE à l'adresse suivante : http://www.csep.ca/francais/ view.asp? $x=804$. Cet article se propose de présenter un abrégé de ce processus de même que les directives ellesmêmes.

\section{Méthodologie}

L'élaboration entière des directives a été conforme au cadre de travail expliqué en détail par Tremblay et Haskell (2012). En bref, le processus d'élaboration des directives comprend 15 stades : constituer une équipe-pilote, instaurer des procédures d'évaluation du processus, former un comité de recherche et d'élaboration des directives, harmoniser les directives aux niveaux interjuridictionnel et international, réaliser une analyse documentaire systématique, interpréter les résultats, identifier les problématiques de recherche, impliquer les parties concernées et arriver à un consensus, élaborer une stratégie de transfert du savoir (incluant la traduction, la formulation du message, la stratégie de communication et de diffusion), évaluer et planifier la révision et la mise à jour.

La figure 1 présente sommairement l'ensemble des événements qui ont mené à l'élaboration des premières directives canadiennes en matière d'activité physique à l'intention des enfants âgés de 0 à 4 ans. Pour plus d'information sur les événements et les processus de 2006 à 2010, veuillez consulter les articles suivants : (Tremblay et al. 2007a, 2007b, 2010a, 2011a; CSEP 2009). Après la publication des nouvelles directives en matière d'activité physique à l'intention des enfants d'âge scolaire, des jeunes, des adultes et des personnes âgées (Tremblay et al. 2011a), la SCPE s'est donné comme mandat prioritaire d'élaborer des directives à l'intention des enfants âgés de moins de 5 ans. La SCPE a pris le leadership du projet qu'elle a réalisé en collaboration avec ParticipACTION et le groupe de recherche HALO (Healthy Active Living and Obesity) du Centre hospitalier pour enfants de l'est de l'Ontario. Le projet a bénéficié d'une subvention des Instituts de recherche en santé du Canada pour l'application des connaissances. L'élaboration complète des directives a respecté la Grille II d'évaluation de la qualité des recommandations pour la pratique clinique (AGREE) (Brouwers et al. 2010a, 2010b, 2010c), un outil reconnu internationalement dans l'élaboration de directives qui permet de guider et d'évaluer la rigueur scientifique et la transparence tout au long du processus. Deux consultants en méthodologie de la recherche (SCG, MEK) ont été embauchés pour informer l'équipe-pilote des meilleures pratiques pour l'élaboration des directives et pour la réalisation de l'analyse documentaire.
Un comité de recherche et d'élaboration des directives, constitué des auteurs de la présente étude, avait les ressources humaines et l'expertise pour mener à bien les tâches nécessaires à l'élaboration des directives. Ce comité a contribué par son savoir et ses conseils à la réalisation de l'analyse documentaire systématique, à l'interprétation des résultats, à l'harmonisation des directives aux niveaux interjuridictionnel et international et à l'identification des problématiques de recherche.

L'analyse documentaire systématique avait pour but de relever et d'évaluer toutes les données probantes portant sur la relation entre l'activité physique et les indicateurs de santé au cours de la petite enfance afin de servir à l'élaboration des directives en matière de santé publique. La question de recherche était la suivante : " quelles sont les caractéristiques (fréquence, intensité, durée, type) mesurables de l'activité physique par des méthodes directes et indirectes et pouvant être associées à l'amélioration des indicateurs de la santé chez les enfants de moins de 5 ans? » Par l'analyse documentaire systématique, on a identifié et compilé les meilleures données probantes disponibles au sujet des quantités minimale et optimale d'activité physique nécessaires à une croissance et un développement sains (masse corporelle saine, santé des os et du squelette, développement des habiletés motrices, santé psychosociale, développement cognitif et facteurs de risque de maladie cardiométabolique) chez des nourrissons $(<1$ an), des tout-petits (1-2 ans) et des enfants d'âge préscolaire (3-4 ans). On a aussi inclus les études qui combinaient des observations durant la petite enfance et plus tard dans la vie. La qualité des données probantes dans l'analyse documentaire a été évaluée au moyen de la méthodologie GRADE (Grading of Recommendations Assessment, Development, and Evaluation) (Balshem et al. 2011; Guyatt et al. 2008) et les données ont été enregistrées au registre prospectif international des analyses documentaires systématiques PROSPERO (numéro d'enregistrement : CRD42011001243). Les études pertinentes ont été identifiées dans les bases de données en ligne (Ovid MEDLINE, Ovid EMBASE, Ovid psycINFO, EBSCO SPORTDiscus et Cochrane Central Database), des bibliothèques personnelles et des documents gouvernementaux. Seules les études de haute qualité (expérimentale, cas-témoin, cohorte prospective) ont été incluses dans l'analyse documentaire. Nous avons priorisé les indicateurs de santé les plus pertinents par groupe d'âge sur le plan de la croissance et du développement (tableau 1). Pour plus d'information sur l'analyse documentaire systématique, veuillez consulter Timmons et al., sous presse.

\section{Réunion de concertation}

En décembre 2011, le comité de recherche et d'élaboration des directives s'est réuni durant 1,5 jour pour ébaucher les recommandations. Ces recommandations étaient basées sur les données probantes présentées dans l'analyse documentaire systématique décrite précédemment. Les participants ont aussi reçu des renseignements généraux et notamment des documents utiles comme les directives du Royaume-Uni et de l'Australie, les précédents documents sur les directives canadiennes en matière d'activité physique et des explications concernant les outils méthodologiques GRADE et AGREE II. La réunion de concertation a permis d'en arriver à un préambule pour expliquer les directives et à l'ébauche 
Fig. 1. Aperçu chronologique des événements principaux dans l'élaboration des Directives canadiennes en matière d'activité physique pour la petite enfance (enfants âgés de 0 à 4 ans).

\section{Directives canadiennes en matière d'activité physique pour la petite enfance (enfants âgés de 0 à 4 ans)}

\section{CHRONOLOGIE}

NOVEMBRE 2006: Groupe de réflexion de la SCPE, Halifax (Nouvelle-Écosse)

DÉCEMBRE 2006: Formation du comité d'orientation de la SCPE au sujet des directives en matière d'activité physique

MARS 2007: Journées de réflexion sur la recherche, Kananaskis (Alberta)

Discussion au sujet des douze analyses documentaires (incluant celle sur la petite enfance) et des articles d'introduction et de conclusion

NOVEMBRE 2007: Publication des articles de fonddans la revue Physiologie appliquée, nutrition et métabolisme (PANM) (32: S2). Assemblée générale annuelle de la SCPE 2007. Analyse documentaire des niveaux d'activité physique de la petite enfance (Timmons et al. Physical activity for preschool children - How much and how?)

JANVIER 2009: Conférence de concertation internationale au sujet des directives en matière d'activité physique, Kananaskis (Alberta)

Le groupe de la petite enfance (0-4 ans) est ciblé comme groupe nécessitant des directives pour combler des lacunes en matière de recommandations

SEPTEMBRE 2010: Identification par l'Agence de santé publique du Canada de la petite enfance comme groupe nécessitant des directives pour combler des lacunes en matière de recommandations et consultation en ligne et en personne par la SCPE auprès des intervenants concernés

OCTOBRE 2010: Formation du groupe de travail sur les directives pour la petite enfance

FÉVRIER 2011: Subvention sur la synthèse des connaissances accordée par les Instituts de recherche en santé du Canada au groupe de recherche HALO (Healthy Active Living and Obesity) pour réaliser une analyse documentaire systématique portant sur l'activité physique et les indicateurs de santé durant la petite enfance

MARS 2011: Formulation des questions pour l'analyse documentaire, Toronto (Ontario)

De façon concourante, le groupe HALO et la SCPE commencent à travailler sur les directives pour la petite enfance

DÉCEMBRE 2011: Conférence de concertation internationale, Toronto (Ontario)

Discussion au tour des résultats de l'analyse documentaire, ébauche des directives en matière d'activité physique pour la petite enfance, consultation en ligne auprès des intervenants concernés au sujet du libellé de l'ébauche des directives

JANVIER 2012: Réunion post-consultation des intervenants concernés sur le libellé, Ottawa (Ontario)

Finalisation du libellé des directives en matière d'activité physique, ébauche des feuillets d'information et des documents de communication

FÉVRIER 2012: Traduction en français des directives, des feuillets d'information et de l'article méthodologique

\section{MARS 2012: Présentation des directives aux Canadiennes et aux Canadiens}

Publication en français et en anglais de l'article méthodologique dans la revue PANM; soumission pour publication de I'analyse documentaire systématique; publication par la SCPE du rapport AGREE II

des directives. Ces ébauches de directives ont été acheminées pour commentaires et suggestions à des intervenants concernés.

\section{Participation des intervenants concernés}

Tout au long du processus d'élaboration des directives, un bon nombre d'intervenants concernés se sont mis à la tâche : 
Tableau 1. Classement a priori des indicateurs de santé par tranche d'âge tel qu'établi par consensus au sein du comité de recherche et d'élaboration des directives.

\begin{tabular}{|c|c|c|c|}
\hline Indicateurs de santé & Nourrisson $(<1$ an $)$ & Tout-petit (1-2 ans) & Préscolaire (3-4 ans) \\
\hline Adiposité (p. ex., surpoids, obésité, IMC) & Critique & Critique & Critique \\
\hline Os (p. ex., santé des os et du squelette) & Sans importance & Important & Critique \\
\hline $\begin{array}{l}\text { Développement moteur (p. ex., mouvements globaux, } \\
\text { locomotion/contrôle d'un objet) }\end{array}$ & Critique & Critique & Critique \\
\hline $\begin{array}{l}\text { Santé psychosociale (p. ex., autoefficacité, estime de soi, } \\
\text { comportement social positif, tempérament, agression, } \\
\text { fonctionnement social) }\end{array}$ & Sans importance & Critique & Critique \\
\hline $\begin{array}{l}\text { Développement cognitif (p. ex., développement langagier, } \\
\text { attention) }\end{array}$ & Important & Important & Critique \\
\hline $\begin{array}{l}\text { Santé cardiométabolique (p. ex., pression sanguine, } \\
\text { insulinorésistance, lipides sanguins) }\end{array}$ & Sans importance & Sans importance & Important \\
\hline Risques (blessure) & S.O. & S.O. & S.O. \\
\hline
\end{tabular}

Note: Le comité de recherche et d'élaboration des directives a classé les résultats en fonction du degré d'importance de l'indicateur de santé : critique, important mais non critique ou sans importance. Au moment de la recherche des données probantes et de leur bilan, le comité a mis l'accent sur les indicateurs dits importants et critiques. Le classement a été fait au moyen de l'outil GRADE (Guyatt et al. 2011).

des scientifiques, des élaborateurs de lignes directrices et des utilisateurs potentiels du guide. Les scientifiques ont travaillé à la formulation des questions de recherche, à l'interprétation des données probantes et à la rédaction de l'ébauche des directives; ils ont aussi participé à la consultation, à la rédaction de cet article et à la finalisation de l'analyse documentaire systématique. Le comité de recherche et d'élaboration des directives comprenait aussi des représentants impliqués dans l'élaboration des directives en matière d'activité physique pour la petite enfance en Australie (ADO) et au Royaume-Uni (JJR), des professionnels de la santé et des utilisateurs finaux de ces directives. À la lumière des données probantes présentées dans l'analyse documentaire et des documents de préparation des directives rédigés à l'occasion de la réunion de concertation de décembre 2011, nous avons aussi recueilli les suggestions et les commentaires provenant des intervenants intéressés dans la promotion de l'activité physique et de la santé de la petite enfance, dont des experts de contenu nationaux et internationaux, des professionnels de la santé, des délégués des organismes gouvernementaux et non gouvernementaux, des enseignants, des fournisseurs de soins et des parents. On a aussi demandé à ces intervenants de partager l'enquête de la SCPE avec leurs pairs et leurs collègues afin d'élargir la consultation de base.

La consultation s'est terminée par une série de questions posées en ligne en décembre 2011. Le questionnaire placé en ligne par la SCPE comportait 12 questions sur le libellé et l'approbation des directives suggérées en matière d'activité physique et sur le préambule. On a aussi sollicité des commentaires écrits de la part des répondants en leur promettant un exemplaire des directives mises à jour, une fois la consultation terminée. En décembre 2011, le comité de recherche et d'élaboration des directives s'est réuni à nouveau pour discuter des préoccupations et analyser les commentaires soulevés lors de la consultation des intervenants concernés et pour ajuster le préambule et les directives en conséquence. Cet article présente les directives finales.

\section{Résultats}

\section{Analyse documentaire systématique}

Tous les détails de l'analyse documentaire systématique sont publiés dans un autre article (Timmons et al., sous presse). La requête a relevé 11222 articles (7872 après avoir éliminé les duplicatas); 28 études distinctes couvrant 22 articles répondaient aux critères d'inclusion (certaines études traitaient de plus d'un indicateur de santé et englobaient plus d'un groupe d'âge). Parmi les études distinctes retenues, voici le nombre d'articles traitant de chacun des résultats d'intérêt pour nous : adiposité $(n=11)$, santé des os et du squelette $(n=2)$, développement des habiletés motrices $(n=4)$, santé psychosociale $(n=3)$, développement cognitif $(n=1)$ et les facteurs de risque de maladie cardiométabolique $(n=3)$. Par groupe d'âge maintenant : 5 études distinctes traitent de la santé chez les nourrissons, 2 chez les tout-petits et 11 chez les enfants d'âge préscolaire. La qualité des données probantes a été évaluée conformément à GRADE (Balshem et al. 2011). Chez les nourrissons, la qualité des données probantes est de faible à modérée pour ce qui est de la relation positive entre l'augmentation de la pratique de l'activité physique et une amélioration des mesures d'adiposité, de développement moteur et de développement cognitif. Chez les tout-petits, la qualité des données probantes est modérée pour ce qui est de la relation positive entre l'augmentation de la pratique de l'activité physique et la santé des os et du squelette. Chez les enfants d'âge préscolaire, la qualité des données probantes est de faible à élevée pour ce qui est de la relation entre l'augmentation de la pratique de l'activité physique et une amélioration des mesures d'adiposité, de développement moteur, de santé psychosociale et des indicateurs de santé cardiométabolique. En résumé, l'analyse documentaire systématique révèle que l'augmentation de la pratique de l'activité physique est associée avec une amélioration des mesures d'adiposité, de développement moteur et cognitif, de santé psychosociale et des indicateurs de santé cardiométabolique durant la petite enfance. Aucune étude n'a porté spécifiquement sur les risques associés à l'augmentation de la pratique de l'activité physique de ce groupe d'âge et selon le groupe d'experts travaillant sur les directives, les avantages potentiels surpassent de loin les risques potentiels associés à l'augmentation de la pratique d'une activité physique.

Les études répertoriées dans l'analyse documentaire systématique ne contenaient pas de données spécifiques en ce 
qui concerne la quantité d'activité physique suffisante à la santé optimale de la petite enfance. En l'absence de telles données probantes, les résultats de l'analyse documentaire sont enrichis par la concertation des experts, l'harmonisation au plan international et l'avis des intervenants concernés au sujet de l'élaboration de ces directives.

\section{Résultats des consultations}

Neuf cent vingt-cinq intervenants concernés ont répondu au questionnaire en ligne et 212 ont ajouté des commentaires et suggestions. Le comité de recherche et d'élaboration des directives a analysé les résultats de la consultation en ligne. Globalement, $95 \%$ des répondants ont dit être « totalement d'accord » et « d'accord » au sujet du préambule et des directives. Compte tenu de la nature de la consultation (sondage par cumul), il est impossible de calculer un taux de réponse au questionnaire en ligne. Le résumé de ces résultats est présenté à l'adresse suivante : http://www.csep.ca/english/ view.asp? $x=879$. À la suite de l'évaluation par les pairs, nous avons apporté des correctifs mineurs d'ordre éditorial au préambule et aux directives qui ne modifient pas les recommandations élaborées par consensus. Tous les membres du comité d'élaboration des directives sont d'accord avec la version finale présentée dans ce document.

\section{Directives finales}

Les directives canadiennes en matière d'activité physique pour la petite enfance (enfants âgés de 0 à 4 ans) sont présentées ci-après.

\section{Préambule}

Ces directives s'appliquent à tous les nourrissons (âgés de $<1$ an), aux tout-petits (âgés de 2-3 ans) et aux enfants d'âge préscolaire (âgés de 3-4 ans) vraisemblablement en santé sans égard au sexe, à la race, à l'origine ethnique ou au statut socioéconomique familial. Les parents et les fournisseurs de soins devraient encourager les nourrissons, les tout-petits et les enfants d'âge préscolaire à participer à une variété d'activités physiques amusantes et sécuritaires qui favorisent une croissance et un développement sains, sont adaptées à leur âge, et se produisent en contexte familial, de service de garde, scolaire ou communautaire.

Les nourrissons devraient être physiquement actifs chaque jour dans le cadre d'activités intérieures et extérieures supervisées. Les activités peuvent comprendre : passer du temps sur le ventre, atteindre et saisir des objets, pousser et tirer, et ramper.

Les enfants âgés de moins de 5 ans devraient être physiquement actifs chaque jour dans le cadre de jeux, de sports, de déplacements, de loisirs et de périodes d'éducation physique. Pour les nourrissons et les enfants sédentaires, faire de l'activité physique, même à un niveau inférieur à celui recommandé, peut entraîner certains bienfaits sur le plan de la santé.

Suivre ces directives en matière d'activité physique peut améliorer la motricité, la composition corporelle ainsi que des aspects liés à la santé métabolique et au développement social. Les avantages potentiels surpassent de loin les risques potentiels associés à la pratique d'une activité physique.

Ces directives peuvent convenir à des nourrissons, à des tout-petits et à des enfants d'âge préscolaire aux prises avec une incapacité ou un trouble médical. Toutefois, leurs parents ou les personnes qui en prennent soin devraient consulter un professionnel de la santé afin de connaître le type et la quantité d'activité physique qui leur conviennent.

Cette recommandation attache beaucoup d'importance aux avantages et aux bienfaits associés à l'activité physique qui s'accumulent tout au long de la vie. Elle prend également en considération le fait que les spécialistes préfèrent disposer de lignes directrices à l'intention des jeunes enfants dans ce domaine. De plus, elle tient compte de l'importance d'établir des cibles à des fins de surveillance. On a pris en compte l'avis des experts et on s'est inspiré de directives internationales pour compléter les données probantes ayant servi à l'élaboration des directives.

Pour des directives sur la réduction des comportements sédentaires, veuillez consulter les directives canadiennes en matière de comportement sédentaire : www.csep.ca/guidelines.

\section{Directives}

Pour favoriser une croissance et un développement sains :

- Les nourrissons (âgés de $<1$ an) devraient être physiquement actifs plusieurs fois par jour - particulièrement par l'entremise de jeux interactifs au sol.

- Les tout-petits (âgés de 1-2 ans) et les enfants d'âge préscolaire (âgés de 3-4 ans) devraient être physiquement actifs chaque jour, quelle que soit l'intensité, pendant au moins $180 \mathrm{~min}(3 \mathrm{~h})$ réparties sur toute la journée. Cela devrait comprendre :

- Une variété d'activités dans divers environnements;

- Des activités qui permettent de développer les habiletés motrices;

- Une progression vers au moins 60 min de jeu actif à l'âge de 5 ans.

S'adonner chaque jour à encore plus d'activité physique entraîne plus de bienfaits.

\section{Discussion}

Cet article présente les premières Directives canadiennes en matière d'activité physique pour la petite enfance (enfants âgés de 0 à 4 ans). Ces directives ont été élaborées au moyen d'un processus rigoureux, à la lumière des meilleures données scientifiques probantes et à la suite d'une vaste consultation auprès d'experts et d'intervenants concernés par ce projet. Les directives sont basées sur des données scientifiques probantes et, en l'absence de telles données, sur des directives dans d'autres domaines d'application (American Academy of Pediatrics 2006; Australian Government 2010; Canadian Pediatric Society, Healthy Active Living Committee 2002; Hagan et al. 2008; National Association for Sport and Physical Education 2009; Start Active Stay Active 2011), sur le consensus au sein du comité de recherche et d'élaboration des directives et sur les commentaires reçus des intervenants concernés lors de la consultation. Le tableau 2 présente un aperçu des directives en matière d'activité physique pour la petite enfance provenant d'autres domaines d'application. Pour plus d'information sur le processus d'élaboration des Directives canadiennes en matière 
Tableau 2. Résumé des directives et recommandations en matière d'activité physique pour la petite enfance, et ce, dans d'autres domaines d'application.

\begin{tabular}{|c|c|}
\hline Pays (références) & Directives en matière d'activité physique \\
\hline $\begin{array}{l}\text { Etats-Unis (American Academy } \\
\text { of Pediatrics 2006) }\end{array}$ & $\begin{array}{l}\text { Nourrissons et tout-petits (naissance - } \mathbf{3} \text { ans) } \\
\text { - Il n'y a pas assez de données probantes pour recommander des programmes d'exer- } \\
\text { cices physiques à l'intention des nourrissons et des tout-petits à des fins de promotion } \\
\text { d'une plus grande pratique de l'activité physique. } \\
\text { - On encourage les parents à procurer à leur enfant un environnement de jeu sécuritaire, } \\
\text { enrichissant et structuré de manière suffisante. } \\
\text { - On devrait donner aux nourrissons et aux tout-petits l'occasion de s'amuser en faisant } \\
\text { de l'activité physique à l'extérieur et d'explorer librement ce milieu sous la respons- } \\
\text { abilité d'un fournisseur de soins adulte. }\end{array}$ \\
\hline
\end{tabular}

Enfants d'âge préscolaire (4-6 ans)

- On devrait encourager le jeu libre en mettant l'accent sur le plaisir, la bonne humeur, l'exploration et l'expérimentation en ayant comme souci la dimension sécuritaire et une supervision appropriée.

- Les enfants d'âge préscolaire devraient participer à des jeux non organisés, de préférence sur des surfaces planes avec quelques variables et des directives présentées sous forme de démonstration.

- Parmi les activités suggérées, notons la course, la natation, les culbutes, les lancers et les attrapés.

- Les enfants d'âge préscolaire devraient commencer à marcher sur des distances acceptables en compagnie de membres de la famille.

Australie (Australian

Government 2010)

Canada (Canadian Pediatric Society, Healthy Active Living Committee 2002)

Etats-Unis (National Association for Sport and Physical Education 2009)
Nourrissons (0-1 an)

- Pour le développement sain des nourrissons, on devrait le plus tôt possible les inciter à faire de l'activité physique, notamment à jouer au sol sous supervision dans un contexte sécuritaire.

Tout-petits (1-3 ans) et enfants d'âge préscolaire (3-5 ans)

- Les tout-petits et les enfants d'âge préscolaire devraient être physiquement actifs tous les jours durant au moins $3 \mathrm{~h}$, réparties sur toute la journée.

Les médecins et les professionnels de la santé devraient faire la promotion d'une vie active saine auprès de tous les membres de la famille en :

- Invitant les enfants et les adolescents à augmenter le temps consacré aux activités physiques et sportives d'au moins 30 min par jour incluant au moins 10 min d'activités physiques d'intensité élevée; cette intensité contribue à élever le rythme cardiaque, la fréquence respiratoire et la température corporelle. Pour des bénéfices supplémentaires, ils devraient faire le bilan chaque mois et augmenter graduellement leurs efforts. Une fois atteint l'objectif d'ajouter 30 min aux activités courantes, ils devraient redéfinir leurs objectifs et consacrer plus de temps notamment à des enjeux plus importants, soit divers exercices de mise en charge intégrés aux activités sportives et récréatives, aux tâches quotidiennes, au travail, aux déplacements, aux exercices planifiés et dans les classes d'éducation physique. Pour accroître l'adhésion à la pratique de l'activité physique, les activités devraient être amusantes et non structurées.

- Conseillant aux parents d'inscrire leurs enfants en âge à des activités récréatives et sportives saines sur le plan de la croissance et du développement.

Nourrissons (naissance - 12 moins)

- Les fournisseurs de soin devraient interagir avec les nourrissons au moyen d'activités physiques quotidiennes conçues pour explorer le mouvement et l'environnement.

- Les fournisseurs de soin devraient placer les nourrissons dans des contextes de jeu actif et stimulant sur le plan du mouvement, et ce, au cours de brèves périodes plusieurs fois par jour.

- Les activités physiques proposées aux nourrissons devraient favoriser le développement d'aptitudes motrices.

- On devrait placer les nourrissons dans un milieu qui répond ou dépasse les normes de sécurité, et ce, pour la réalisation des activités sollicitant ses gros muscles.

- Les responsables du bien-être des nourrissons doivent comprendre l'importance de l'activité physique et faire la promotion du développement moteur en créant des occasions de s'adonner à des activités physiques structurées ou non.

Tout-petits (12-36 moins)

- Les tout-petits devraient faire au moins 30 min d'activité physique structurée tous les jours. 
Tableau 2 (suite en fin).

\begin{tabular}{|c|c|}
\hline Pays (références) & Directives en matière d'activité physique \\
\hline & $\begin{array}{l}\text { - Les tout-petits devraient consacrer tous les jours au moins } 60 \text { min - et même quelques } \\
\text { heures - à des activités physiques non structurées et ne devraient pas être sédentaires } \\
\text { durant plus de } 60 \text { min à la fois, sauf en période de sommeil. } \\
\text { - On devrait procurer aux tout-petits de multiples occasions de développer les aptitudes } \\
\text { motrices qui serviront d'assise à leurs futures habiletés motrices et à la pratique de } \\
\text { l'activité physique au cours des années à venir. } \\
\text { - Les tout-petits devraient avoir accès à des espaces intérieurs et extérieurs pour la réa- } \\
\text { lisation d'activités sollicitant leurs grosses masses musculaires et ces espaces devraient } \\
\text { présenter ou dépasser les normes de sécurité. } \\
\text { - Les responsables du bien-être des tout-petits doivent comprendre l'importance de l'ac- } \\
\text { tivité physique et faire la promotion du développement moteur en créant des occasions } \\
\text { d'expérimenter des mouvements et de s'adonner à des activités physiques structurées } \\
\text { ou non. } \\
\text { Enfants d'âge préscolaire (3-5 ans) } \\
\text { - Les enfants d'âge préscolaire devraient faire au moins } 60 \text { min d'activité physique } \\
\text { structurée tous les jours. } \\
\text { - Les enfants d'âge préscolaire devraient consacrer tous les jours au moins } 60 \text { min - et } \\
\text { même quelques heures - à des activités physiques non structurées et ne devraient pas } \\
\text { être sédentaires durant plus de } 60 \text { min à la fois, sauf en période de sommeil. } \\
\text { - On devrait inciter les enfants d'âge préscolaire à développer leurs aptitudes motrices } \\
\text { fondamentales qui serviront d'assise à leurs futures habiletés motrices et à la pratique } \\
\text { de l'activité physique au cours des années à venir. } \\
\text { - Les enfants d'âge préscolaire devraient avoir accès à des espaces intérieurs et extér- } \\
\text { ieurs pour la réalisation d'activités sollicitant leurs grosses masses musculaires et ces } \\
\text { espaces devraient présenter ou dépasser les normes de sécurité. } \\
\text { - Les fournisseurs de soins et les parents, responsables de la santé et du bien-être des } \\
\text { enfants d'âge préscolaire, doivent comprendre l'importance de l'activité physique et } \\
\text { faire la promotion du développement moteur en créant des occasions de s'adonner à } \\
\text { des activités physiques structurées ou non. }\end{array}$ \\
\hline $\begin{array}{l}\text { Royaume-Uni (Start Active Stay } \\
\text { Active 2011) }\end{array}$ & $\begin{array}{l}\text { Nourrissons qui ne marchent pas encore } \\
\text { - On devrait encourager la pratique de l'activité physique dès la naissance, notamment } \\
\text { par des jeux au sol et dans l'eau dans un contexte sécuritaire. } \\
\text { Tout-petits (moins de } \mathbf{5} \text { ans) qui sont capables de marcher } \\
\text { - Les enfants d'âge préscolaire qui sont capables de marcher sans aide devraient être } \\
\text { physiquement actifs durant au moins } 180 \text { min }(3 \text { h) réparties sur toute la journée. } \\
\text { - On devrait prendre en compte les capacités physiques et mentales lorsqu'il s'agit d'in- } \\
\text { terpréter les directives. }\end{array}$ \\
\hline
\end{tabular}

Note : Les directives en matière de comportement sédentaire durant la petite enfance ne sont pas énumérées ici; on peut les consulter dans l'article sur les Directives canadiennes en matière de comportement sédentaire pour la petite enfance (Tremblay et al. 2012).

d'activité physique, veuillez consulter : http://www.csep.ca/ Francais/view.asp? $\mathrm{x}=804$.

La forme et le format des directives et des recommandations des divers domaines d'application diffèrent substantiellement (tableau 2). Ces nouvelles directives canadiennes empruntent la forme et le format utilisés par la SCPE pour ses autres directives (Tremblay et al. 2011a, 2011b). Dans l'ensemble, les nouvelles directives canadiennes s'harmonisent aux directives présentées dans le tableau 2; cependant, il y a quelques différences dignes de mention. Premièrement, les groupes d'âge varient d'un domaine d'application à l'autre. Les groupes d'âge dans les directives canadiennes sont définis en mode continu, de la naissance à 4 ans (petite enfance), de 5 à 11 ans (enfants), de 12 à 17 ans (jeunes), de 18 à 64 ans (adultes) et de 65 ans et plus (aînés) et en fonction des étapes de la vie : entrée à l'école (5 ans), fin des études secondaires (18 ans) et début de la retraite (65 ans). Deuxièmement, la terminologie utilisée varie d'un domaine d'application à l'autre (p. ex., petite enfance, jeunes enfants, enfants d'âge préscolaire). La terminologie utilisée dans les directives canadiennes a été définie par consensus d'experts et au moyen des résultats du sondage en ligne réalisé auprès des parties concernées. Troisièmement, les directives de l'Association nationale américaine des sports et de l'éducation physique (NASPE 2009) incluent des recommandations pour l'activité physique structurée ou non. Même si on a discuté en détail de cet aspect au cours du processus d'élaboration des directives canadiennes, on s'est abstenu d'inclure une telle division en l'absence de données probantes à cette fin. Cependant, les documents de promotion des directives à l'intention des personnes qui prennent soin des enfants contiendront des propositions d'activité physique structurée ou non permettant de se conformer aux directives. En dernier lieu, la Société canadienne de pédiatrie travaille actuellement à la mise à jour de ses directives à la lumière des nouvelles données probantes et, après sa publication en 2012, elle retirera sa déclaration précédente (2002).

Les nouvelles directives sont basées sur des données probantes; elles sont réalistes et réalisables, approuvées par un grand nombre d'experts et s'arriment aux directives d'autres 
organismes. Les directives en matière d'activité physique à l'intention des enfants âgés de 0 à 4 ans sont conformes au message global inclus dans les directives canadiennes en vigueur en matière d'activité physique : " plus, c'est mieux »; dès lors, les nouvelles directives devraient être considérées comme une cible initiale.

La principale différence entre les directives à l'intention des enfants d'âge scolaire et celles pour la petite enfance réside dans l'intensité de l'activité physique. Les directives pour la petite enfance recommandent aux tout-petits et aux enfants d'âge préscolaire au moins 180 min d'activité physique quelle qu'en soit l'intensité alors que les directives à l'intention des enfants d'âge scolaire recommandent au moins 60 min d'activité d'intensité modérée à intense, et ce, tous les jours (Tremblay et al. 2011a). On pourrait en déduire que les recommandations diminuent de $180 \mathrm{~min}$ à $60 \mathrm{~min}$ quand les enfants commencent à aller à l'école; ce n'est pas le cas. De fait, la recommandation d'augmenter graduellement dans la petite enfance la durée de pratique pour atteindre 60 min de jeu actif (intensité modérée à intense) à l'âge de 5 ans est telle que les directives respectives se confondent tout en gardant le message et le contexte propres à chaque groupe d'âge. Pour concilier la différence apparente en ce qui concerne la durée, les enfants d'âge scolaire devraient se conformer à la recommandation de $60 \mathrm{~min}$, et ce, en sus des activités « non planifiées réalisées dans le cadre de la vie de tous les jours » (Tremblay et al. 2011a); cet aspect est inclus dans la recommandation pour la petite enfance.

\section{Diffusion et mise en æuvre}

Le processus d'élaboration des directives en matière d'activité physique pour la petite enfance est présenté dans cet article. Le rapport complet sur les recommandations pour la pratique clinique et l'évaluation au moyen d'AGREE II sont accessibles au public à l'adresse suivante : http://www.csep. ca/Francais/view.asp? $\mathrm{x}=804$. De plus, la démarche méthodologique, les analyses documentaires systématiques et les recommandations finales ont été et seront présentées au cours de réunions scientifiques et sont disponibles sur le site de la SCPE : www.csep.ca.

Ces nouvelles directives sont approuvées, soutenues et publiées par la SCPE, ParticipACTION, les partenaires fédéraux, provinciaux et territoriaux, les groupes de personnes concernées par la pratique de l'activité physique et par des personnes dédiées à la cause. Le processus de diffusion et de mise en ouvre est dicté par un ensemble de recommandations proposées par un comité d'experts comprenant les auteurs des directives, des spécialistes en marketing et en communication dans le domaine de la santé et des chercheurs en modification comportementale en matière de santé. Les étapes franchies pour l'élaboration de ces recommandations ont été conformes au rigoureux processus utilisé pour l'élaboration des directives en matière d'activité physique. Le processus utilisé pour définir et élaborer le véhicule des recommandations accompagnant les nouvelles directives est publié dans d'autres documents (Latimer et al. 2010; Rhodes et Pfaeffli 2010; www.csep.ca). Du fait qu'il faut du temps et des ressources pour élaborer les stratégies de communication et de diffusion des documents au public, la SCPE, en collaboration avec les intervenants concernés, continuera au cours des prochains mois (et années) son travail pour combler les lacunes existantes actuellement. Ces documents comprendront des feuillets d'information à l'intention des professionnels de la santé et des fournisseurs de soins (Annexe A), des affiches, des vignettes, des témoignages inspirants, des webinaires et des livres numériques. Tous les documents préparés par la SCPE seront accessibles sur le site Internet de la SCPE et, le cas échéant, seront publicisés par des partenaires et des intervenants dédiés à la cause.

\section{Mise à jour des directives}

La mise à jour de ces nouvelles directives sera importante et nécessaire afin que les directives restent pertinentes à la lumière des données nouvelles. La tâche immense associée à la mise à jour des analyses documentaires rend difficile une mise à jour simultanée de toutes les tranches d'âge. Alors, on recommande une mise à jour cyclique des directives, soit tous les 5 ans. Toutefois, si des données nouvelles se présentent entretemps, les responsables effectueront les mises à jour dans un délai plus court. On recommande aussi de coordonner les mises à jour avec les autres domaines d'application et pays pour éviter le dédoublement des tâches et harmoniser les directives en matière d'activité physique.

\section{Surveillance}

Au Canada, il y a très peu d'activité de surveillance en ce qui concerne l'activité physique dans la petite enfance et particulièrement auprès d'échantillons nationaux représentatifs. L'enquête longitudinale nationale sur les enfants et les jeunes (ELNEJ, Statistiques Canada, http://www.statcan.gc.ca/imdbbmdi/instrument/4450_Q2_V7-eng.pdf) a collecté des données au sujet des comportements en matière d'activité physique chez les enfants et les jeunes âgés de 0 à 17 ans, mais ne le fait plus. L'enquête canadienne sur les mesures de la santé (ECMS, Statistiques Canada, http://www.statcan.gc.ca/cgi-bin/ $\mathrm{imdb} / \mathrm{p} 2 \mathrm{SV}$.pl? Function $=$ getSurvey $\&$ SDDS $=5071 \&$ lang $=$ en $\& d b=i m d b \& a d m=8 \& d i s=2)$ cycle $2(2009-2011)$ a utilisé des accéléromètres pour recueillir des données objectives sur l'activité physique des enfants âgés de 3 et 4 ans (en plus d'enfants plus âgés). Les données de l'ECMS vont permettre l'évaluation directe d'un échantillon d'enfants représentatif au plan national et vont nous renseigner sur le nombre d'enfants d'âge préscolaire se conformant aux directives. Les prochaines phases du Sondage indicateur de l'activité physique (SAP, Institut canadien de la recherche sur la condition physique et le mode de vie, http://www.cflri.ca/pub_page/105) devront s'accommoder des observations rapportées par les parents en ce qui concerne les comportements en matière d'activité physique de leurs enfants âgés de 0 à 4 ans, même si la validité de cette approche n'est pas clairement établie.

Pour des exemples, en ce qui concerne spécifiquement les activités de surveillance de l'activité physique durant la petite enfance, veuillez consulter le bulletin Jeunes en forme Canada (Active Healthy Kids Canada 2008, 2009, 2010, 2011). La surveillance des comportements d'activité physique au Canada présente des limitations et des lacunes claires (Katzmarzyk et Tremblay 2007). Il faut consacrer des efforts supplémentaires pour observer les comportements de la petite enfance en matière d'activité physique. En espérant que la publication de ces directives donne l'élan initial. 


\section{Prochaines études}

Les études dans ce domaine n'en sont qu'à leur début; il faudra donc beaucoup plus d'études pour comprendre clairement la relation entre les caractéristiques de l'activité physique (fréquence, intensité, durée, type) et les résultats au plan de la santé dans la petite enfance. Ces directives ont été élaborées à la lumière des meilleures données probantes; quoique les études ne soient pas exhaustives, le besoin manifesté par les Canadiennes et les Canadiens pour des directives en matière de vie active saine pour la petite enfance nous a incités grandement à élaborer maintenant ces directives. Les auteurs de cette étude souhaitent que ces directives à l'intention de cette tranche d'âge stimulent la recherche, même celle qui la mette en doute. Il faut faire des études sur les nourrissons, les tout-petits et les enfants d'âge préscolaire afin de :

- Comprendre la relation entre la fréquence, l'intensité, la durée et le type d'activité physique et les indicateurs de la santé et leurs améliorations.

- Évaluer l'efficacité et l'aspect sécuritaire de ces directives pour des enfants présentant des besoins particuliers (maladie chronique, incapacité).

- Comprendre la relation et les effets de l'activité physique de diverses intensités et les comportements sédentaires (par ex., rester assis ou regarder la télévision) de diverses durées.

- Comprendre les moyens les plus efficaces pour communiquer de nouvelles directives aux intermédiaires et au public en général.

- Observer les effets indésirables associés aux niveaux recommandés de pratique de l'activité physique dans cet article.

- Déterminer les meilleures méthodes et mesures de l'activité physique à utiliser auprès de la petite enfance.

- Identifier les meilleurs indicateurs de santé (ou des substituts) sensibles à l'activité physique de la petite enfance.

- Utiliser de rigoureux protocoles de recherche

- Comportant des échantillons représentatifs de la population dans une étude longitudinale structurée, des mesures directes (objectives) standardisées de l'activité physique et des résultats au plan de la santé selon l'âge tout en prenant en compte les covariables suivantes : âge, sexe, statut socio-économique et origine ethnique.

- Pour hausser la qualité des essais cliniques aléatoires chez cette tranche d'âge (échantillons plus grands et diversifiés avec des mesures directes et des résultats signifiants sur le plan de la croissance et du développement).

\section{Comportements sédentaires}

Les autres directives ou recommandations en matière d'activité physique pour la petite enfance énumérées au tableau 2 incluent les énoncés au sujet de la diminution des comportements sédentaires, tout comme les précédentes directives canadiennes en matière d'activité physique à l'intention des enfants (Health Canada and the Canadian Society for Exercise Physiology 2002). Cependant, d'après des données probantes récentes, la biologie (et donc des conséquences sur la santé) des comportements sédentaires serait distincte de la biologie de l'activité physique (Tremblay et al. 2010c), nous avons donc décidé d'élaborer des directives distinctes en matière d'activité physique et de comportements sédentaires basées sur des analyses documentaires systématiques distinctes, comme la SCPE l'a fait pour les enfants d'âge scolaire (Tremblay et al. 2011a, 2011b). L'article sur les premières directives en matière de comportements sédentaires pour la petite enfance est aussi inclus dans ce numéro du périodique (Tremblay et al. 2012).

\section{En bref}

Cet article donne un aperçu du processus suivi pour l'élaboration des nouvelles Directives canadiennes en matière d'activité physique pour la petite enfance (enfants âgés de 0 à 4 ans). Ces directives ont été élaborées en collaboration avec de nombreux organismes afin de présenter les meilleures données probantes sur la relation entre l'activité physique et divers indicateurs de la santé. Le comité de recherche et d'élaboration des directives a vu à ce que le processus d'élaboration des directives soit rigoureux, transparent, inclusif et solidement documenté.

\section{Remerciements}

Les auteurs remercient la SCPE pour son rôle de leader dans l'élaboration des nouvelles directives en matière d'activité physique, ParticipACTION pour son rôle de partenaire de premier plan dans la diffusion de ces directives et le groupe de recherche HALO pour son rôle de leader du processus scientifique d'élaboration des directives. Le financement de l'analyse documentaire systématique à la base de ces directives est venu des IRSC (PAC-111612) grâce à son programme Subvention de synthèse, application des connaissances. La SCPE et le groupe de recherche HALO ont octroyé des fonds supplémentaires pour l'élaboration de ces directives. Le point de vue des organismes de financement n'a eu aucun impact sur le contenu et les recommandations apparaissant dans ce document. Nous en profitons pour remercier aussi les intervenants concernés, les partenaires et les participants pour leur contribution à l'élaboration de ces directives grâce à leur implication au sein du comité de recherche et d'élaboration des directives et leur participation aux consultations en ligne. Ian Janssen et Amy Latimer détiennent une chaire de niveau 2 des chaires de recherche du Canada à l'Université Queen's. Brian Timmons détient une bourse des IRSC pour nouveau chercheur. Michelle Kho a reçu du financement des Instituts de recherche en santé du Canada : une bourse de recherche et le prix Bisby. Valérie Carson détient une bourse d'études supérieures (doctorat) du Canada Frederick Banting et Charles Best. Jodie Stearns détient une bourse de maîtrise CGS Joseph-Armand Bombardier du Conseil de recherches en sciences humaines.

\section{Bibliographie}

Active Healthy Kids Canada. 2008. It's time to unplug our kids Report Card on Physical Activity for Children and Youth. Active Healthy Kids Canada. Toronto, Active Healthy Kids Canada.

Active Healthy Kids Canada. 2009. Active kids are fit to learn Report Card on Physical Activity for Children and Youth. Active Healthy Kids Canada. Toronto, Active Healthy Kids Canada.

Active Healthy Kids Canada. 2010. Healthy habits start earlier than you think - Report Card on Physical Activity for Children and 
Youth. Active Healthy Kids Canada. Toronto, Active Healthy Kids Canada.

Active Healthy Kids Canada. 2011. Don't let this be the most physical activity our kids get after school - Report Card on Physical Activity for Children and Youth. Active Healthy Kids Canada. Toronto, Active Healthy Kids Canada.

American Academy of Pediatrics; Council on Sports Medicine and Fitness and Council on School Health. 2006. Active healthy living: prevention of childhood obesity through increased physical activity. Pediatrics, 117(5) : 1834-1842. doi:10.1542/peds.20060472. PMID:16651347.

Australian Government. 2010. Move and Play Every Day. National physical activity recommendations for children 0-5 years. Commonwealth of Australia, Department of Health and Ageing.

Balshem, H., Helfand, M., Schunemann, H.J., Oxman, A.D., Kunz, R., Brozek, J., et al. 2011. GRADE guidelines: 3. Rating the quality of evidence. J. Clin. Epidemiol. 64(4) : 401-406. doi:10. 1016/j.jclinepi.2010.07.015. PMID:21208779.

Brouwers, M.C., Kho, M.E., Browman, G.P., Burgers, J.S., Cluzeau, F., Feder, G., et al.; AGREE Next Steps Consortium. 2010a. AGREE II: Advancing guideline development, reporting and evaluation in health care. CMAJ, 182(18) : E839-E842. doi:10. 1503/cmaj.090449. PMID:20603348.

Brouwers, M.C., Kho, M.E., Browman, G.P., Burgers, J.S., Cluzeau, F., Feder, G., et al.; AGREE Next Steps Consortium. $2010 b$. Development of the AGREE II, part 1: performance, usefulness and areas for improvement. CMAJ, 182(10) : 1045-1052. doi:10. 1503/cmaj.091714. PMID:20513780.

Brouwers, M.C., Kho, M.E., Browman, G.P., Burgers, J.S., Cluzeau, F., Feder, G., et al.; AGREE Next Steps Consortium. 2010c. Development of the AGREE II, part 2: assessment of validity of items and tools to support application. CMAJ, 182(10) : E472E478. doi:10.1503/cmaj.091716. PMID:20513779.

Canadian Pediatric Society, Healthy Active Living Committee. 2002. Healthy active living for children and youth. Paediatr. Child Health (Oxford), 7 : 339-345.

Canning, P.M., Courage, M.L., et Frizzell, L.M. 2004. Prevalence of overweight and obesity in a provincial population of Canadian preschool children. CMAJ, 171(3) : 240-242. doi:10.1503/cmaj. 1040075. PMID:15289421.

Colley, R.C., Garriguet, D., Janssen, I., Craig, C., Clarke, J., et Tremblay, M.S. 2011. Physical activity of Canadian children and youth: Accelerometer results from the 2007-2009 Canadian Health Measures Survey. Health Rep. 22(1) : 15-23. PMID: 21510586.

CSEP. 2009. 2009 Consensus Conference: Advancing the Future of Physical Activity Measurement and Guidelines. Final Report. Canadian Society for Exercise Physiology.

CSEP. 2011. CSEP Physical Activity Guidelines Stakeholder Survey Results and Final Report. Canadian Society for Exercise Physiology.

Guyatt, G.H., Oxman, A.D., Vist, G.E., Kunz, R., Falck-Vitter, Y., Alonso-Coello, P., et Schünemann, H.J.; GRADE Working Group. 2008. GRADE: an emerging consensus on rating quality of evidence and strength of recommendations. BMJ, 336(7650) : 924-926. doi:10.1136/bmj.39489.470347.AD. PMID:18436948.

Guyatt, G.H., Oxman, A.D., Kunz, R., Atkins, D., Brozek, J., Vist, G., et al. 2011. GRADE guidelines 2. Framing the question and deciding on important outcomes. J. Clin. Epidemiol. 64(4) : 395400. doi:10.1016/j.jclinepi.2010.09.012. PMID:21194891.

Hagan, J.F., Jr., Shaw, J.S., et Duncan, P. (Editeurs.) 2008. Bright Futures Guidelines for Health Supervision of Infants, Children, and Adolescents - Third Edition. American Academy of Pediatrics.
Health Canada and the Canadian Society for Exercise Physiology. 2002. Canada's physical activity guide for children. Cat. no. H39611/2002-2E. Minister of Public Works and Government Services Canada, Ottawa, Ont., Canada.

Janssen, I. 2007. Physical activity guidelines for children and youth. [Version française disponible à Appl. Physiol. Nutr. Metab. 32 (S2F) : S122-S135.] Appl. Physiol. Nutr. Metab. 32(S2E) : S109S121. doi:10.1139/H07-109.

Janssen, I., et LeBlanc, A.G. 2010. Systematic review of the health benefits of physical activity and fitness in school-aged children and youth. Int. J. Behav. Nutr. Phys. Act. 7(40) : 1-16. doi:10. 1186/1479-5868-7-40.

Katzmarzyk, P.T., et Tremblay, M.S. 2007. Limitations of Canada's physical activity data: implications for monitoring trends. [Version française disponible à Appl. Physiol. Nutr. Metab. 32(S2F) : S206-S216.] Appl. Physiol. Nutr. Metab. 32(Suppl. 2E) : S185S194. doi:10.1139/H07-113.

Latimer, A., Brawley, L., et Bassett, R. 2010. A systematic review of three approaches for constructing physical activity messages: what messages work and what improvements are needed? Int. J. Behav. Nutr. Phys. Act. 7(36) : 1-17. doi:10.1186/1479-5868-7-36.

National Association for Sport and Physical Education. 2009. Active Start: A Statement of Physical Activity Guidelines for Children From Birth to Age 5 - Second Edition. AAHPERD Publications, Oxon Hill, Md., É.-U.

Obeid, J., Nguyen, T., Gabel, L., et Timmons, B.W. 2011. Physical activity in Ontario preschoolers: Prevalence and measurement issues. Appl. Physiol. Nutr. Metab. 36(2) : 291-297. doi:10.1139/ h11-002. PMID:21609292.

Physical Activity Guidelines Advisory Committee. 2008. Physical Activity Guidelines Advisory Committee Report 2008. Department of Health and Human Services, Washington, D.C., É.-U.

Rhodes, R., et Pfaeffli, L. 2010. Mediators of physical activity behaviour change among adult non-clinical populations: a review update. Int. J. Behav. Nutr. Phys. Act. 7 : 37. PMID:20459781.

Shields, M. 2006. Overweight and obesity among children and youth. Health Rep. 17(3) : 27-42. PMID:16981484.

Start Active Stay Active. 2011. Start Active, Stay Active: a report on physical activity for health from the four home countries' Chief Medical Officers. 2011. Royaume-Uni. Disponible au www.dh.gov.uk/en/Publicationsandstatistics/Publications/ PublicationsPolicyAndGuidance/DH_128209 [cité le 9 janvier 2012].

Temple, V.A., Naylor, P.-J., Rhodes, R.E., et Higgins, J.W. 2009. Physical activity of children in family child care. Appl. Physiol. Nutr. Metab. 34(4) : 794-798. doi:10.1139/H09-061. PMID: 19767816.

Timmons, B.W., Naylor, P.J., et Pfeiffer, K. 2007. Physical activity for preschool children - how much and how. [Version française disponible à Appl. Physiol. Nutr. Metab. 32(S2F) : S136-S149.] Appl. Physiol. Nutr. Metab. 32(S2E) : S122-S134. doi:10.1139/ H07-112.

Timmons, B.W., LeBlanc, A.G., Carson, V., Connor Gorber, S., Dillman, C., Janssen, I., et al. Systematic review of the relationship between physical activity and health indicators in the early years (ages 0-4 years). Appl. Physiol. Nutr. Metab., Sous presse.

Tremblay, M.S., et Haskell, W.L. 2012. From science to physical activity guidelines. Dans Physical Activity and Health. Sous la direction de C. Bouchard, S.N. Blair, et W.L. Haskell. 2ième ed. Human Kinetics Publishers, Champaign, Ill., É.-U. p. 359-378.

Tremblay, M.S., et Willms, J.D. 2000. Secular trends in body mass index of Canadian children. CMAJ, 163(11) : 1429-1433; erratum 2001, 164(7) : 970.. PMID:11192647.

Tremblay, M.S., Katzmarzyk, P.T., et Willms, J.D. 2002. Temporal 
trends in overweight and obesity in Canada 1981-1996. Int. J. Obes. Relat. Metab. Disord. 26(4) : 538-543. doi:10.1038/sj.ijo. 0801923. PMID:12075581.

Tremblay, M.S., Shephard, R.J., et Brawley, L. 2007a. Research that informs Canada's physical activity guides: an introduction. [Version française disponible à Appl. Physiol. Nutr. Metab. 32 (S2F) : S1-S9.] Appl. Physiol. Nutr. Metab. 32(Suppl. 2E) : S1S8. doi:10.1139/H07-104.

Tremblay, M.S., Shephard, R.J., Brawley, L., Cameron, C., Craig, C.L., Duggan, M., et al. 2007b. Physical activity guidelines and guides for Canadians: facts and future. [Version française disponible à Appl. Physiol. Nutr. Metab. 32(S2F) : S242-S249.] Appl. Physiol. Nutr. Metab. 32(Suppl. 2E) : S218-S224. doi:10.1139/H07-125.

Tremblay, M.S., Kho, M.E., Tricco, A.C., et Duggan, M. $2010 a$. Process description and evaluation of Canadian Physical Activity Guidelines development. Int. J. Behav. Nutr. Phys. Act. 7: 42. PMID:20459786.

Tremblay, M.S., Shields, M., Laviolette, M., Craig, C.L., Janssen, I., et Connor Gorber, S. 2010b. Fitness of Canadian children and youth: results from the 2007-2009 Canadian Health Measures Survey. Health Rep. 21(1) : 7-20. PMID:20426223.

Tremblay, M.S., Colley, R., Saunders, T.J., Healy, G.N., et Owen, N. 2010c. Physiological and health implications of a sedentary lifestyle. Appl. Physiol. Nutr. Metab. 35(6) : 725-740. doi:10. 1139/H10-079. PMID:21164543.
Tremblay, M.S., Warburton, D.E.R., Janssen, I., Paterson, D.H., Latimer, A.E., Rhodes, R.E., et al. 2011a. New Canadian Physical Activity Guidelines. Appl. Physiol. Nutr. Metab. 36(1) : 36-46, 47-58. doi:10.1139/H11-009. PMID:21326376.

Tremblay, M.S., Leblanc, A.G., Janssen, I., Kho, M.E., Hicks, A., Murumets, K., et al. 2011b. Canadian Sedentary Behaviour Guidelines for Children and Youth. Appl. Physiol. Nutr. Metab. 36(1) : 59-64, 65-71. doi:10.1139/H11-012. PMID:21326378.

Tremblay, M.S., LeBlanc, A.G., Carson, V., Choquette, L., Connor Gorber, S., Dillman, C., et al. 2012. Canadian Sedentary Behaviour Guidelines for the Early Years (aged 0-4 years) Appl. Physiol. Nutr. Metab. 37(2) : 370-380, 381-391. doi:10. 1139/H2012-019.

Tucker, P. 2008. The physical activity levels of preschool-aged children: a systematic review. Early Child. Res. Q. 23(4) : 547558. doi:10.1016/j.ecresq.2008.08.005.

World Health Organization. 2010. Global recommendations on physical activity for health. World Health Organization. Geneva, Switzerland.

\section{Annexe A}

L'annexe est présenté en page suivante. 
Fig. A1. Feuillet d'information sur les Directives canadiennes en matière d'activité physique pour la petite enfance (enfants âgés de 0 à 4 ans).

\section{Directives canadiennes en matière d'activité physique}

\section{POUR LA PETITE ENFANCE DE 0 À 4 ANS}

\section{Directives}

Pour favoriser une croissance et un développement sains :

Les nourrissons (âgés de moins de 1 an) devraient être physiquement actifs plusieurs fois

par jour - particulièrement par l'entremise de jeux interactifs au sol.

Les tout-petits (âgés de 1 à 2 ans) et les enfants d'âge préscolaire (âgés de 3 à 4 ans) devraient faire au moins 180 minutes d'activité physique, peu importe l'intensité, réparties au cours de la journée, y compris :

Une variété d'activités dans divers environnements;

(27) Des activités qui permettent de développer les habiletés motrices;

Une progression vers au moins 60 minutes de jeu actif à l'âge de 5 ans.

S'adonner chaque jour à encore plus d'activité physique entraîne plus de bienfaits.

Pour un nourrisson, être actif signifie :

- Passer du temps sur le ventre

- Atteindre ou saisir des ballons ou d'autres jouets

- Jouer ou rouler sur le sol

- Ramper dans la maison

Pour un tout-petit ou un enfant d'âge préscolaire, être actif signifie :

- Réaliser toute activité qui permet de bouger

- Monter les escaliers et bouger dans la maison

- Jouer dehors et explorer son environnement

- Ramper, marcher rapidement, courir ou danser

Plus les enfants vieillissent, plus ils ont besoin de prendre part à des jeux énergiques, par exemple sautiller, sauter, gambader et faire du vélo.
Être actifs peut aider les jeunes enfants à :

- Maintenir un poids corporel santé

- Améliorer leur motricité

- Améliorer leur condition physique

- Avoir un cœur en santé

- Avoir du plaisir et se sentir bien

- Développer de la confiance en soi

- Améliorer leur capacité d'apprentissage et d'attention

Toutes les activités comptent. Essayez ces trucs pour faire bouger les jeunes enfants :

■ Créer des espaces sécuritaires pour le jeu.

$\checkmark$ Mettre de la musique et apprendre ensemble des chansons. accompagnées de mouvements.
■ S'habiller en fonction de la météo et explorer à l'extérieur.

$\square$ Planifier du temps pour jouer avec d'autres enfants.

$\square$ Se déplacer à pied ou à vélo.

\section{Bouger tous les jours. Bouger ensemble!}

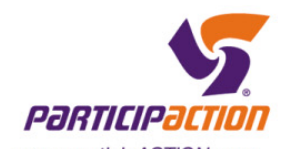

www.participACTION.com

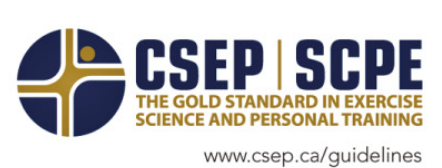

